TRANSACTIONS OF THE

AMERICAN MATHEMATICAL SOCIETY

Volume 362, Number 4, April 2010, Pages 1751-1764

S 0002-9947(09)05169-1

Article electronically published on November 16, 2009

\title{
SQUARES OF MENGER-BOUNDED GROUPS
}

\author{
MICHA£ MACHURA, SAHARON SHELAH, AND BOAZ TSABAN
}

\begin{abstract}
Using a portion of the Continuum Hypothesis, we prove that there is a Menger-bounded (also called o-bounded) subgroup of the Baer-Specker group $\mathbb{Z}^{\mathbb{N}}$, whose square is not Menger-bounded. This settles a major open problem concerning boundedness notions for groups and implies that Mengerbounded groups need not be Scheepers-bounded. This also answers some questions of Banakh, Nickolas, and Sanchis.
\end{abstract}

\section{INTRODUCTION}

Assume that $(G, \cdot)$ is a topological group. For $A, B \subseteq G, A \cdot B$ stands for $\{a \cdot b: a \in A, b \in B\}$ and $a \cdot B$ stands for $\{a \cdot b: b \in B\}$. The following definitions are due, independently, to Okunev and Kočinac.

Definition 1. Assume that $(G, \cdot)$ is a topological group. $G$ is:

(1) Menger-bounded if for each sequence $\left\{U_{n}\right\}_{n \in \mathbb{N}}$ of neighborhoods of the unit, there exist finite sets $F_{n} \subseteq G, n \in \mathbb{N}$, such that $G=\bigcup_{n} F_{n} \cdot U_{n}$.

(2) Scheepers-bounded if for each sequence $\left\{U_{n}\right\}_{n \in \mathbb{N}}$ of neighborhoods of the unit, there exist finite sets $F_{n} \subseteq G, n \in \mathbb{N}$, such that for each finite set $F \subseteq G$, there is $n$ such that $F \subseteq F_{n} \cdot U_{n}$.

(3) Hurewicz-bounded if for each sequence $\left\{U_{n}\right\}_{n \in \mathbb{N}}$ of neighborhoods of the unit, there exist finite sets $F_{n} \subseteq G, n \in \mathbb{N}$, such that for each $g \in G$, $g \in F_{n} \cdot U_{n}$ for all but finitely many $n$.

(4) Rothberger-bounded if for each sequence $\left\{U_{n}\right\}_{n \in \mathbb{N}}$ of neighborhoods of the unit, there exist elements $a_{n} \in G, n \in \mathbb{N}$, such that $G=\bigcup_{n} a_{n} \cdot U_{n}$.

Several instances of these properties were studied in, e.g., [28, 10, 11, 18, 5, 30]. A study from a more general point of view was initiated in [15, 2, 1,. These properties are obtained from the following general topological properties by restricting attention to open covers of the form $\{a \cdot U: a \in G\}$, where $U$ is an open neighborhood of the unit.

Definition 2. Assume that $X$ is a topological space. $X$ has the

(1) Menger property 20] if for each sequence $\left\{\mathcal{U}_{n}\right\}_{n \in \mathbb{N}}$ of open covers of $X$, there exist finite sets $\mathcal{F}_{n} \subseteq \mathcal{U}_{n}, n \in \mathbb{N}$, such that $\bigcup_{n \in \mathbb{N}} \mathcal{F}_{n}$ is a cover of $X$.

Received by the editors May 1, 2007.

2000 Mathematics Subject Classification. Primary 54H11, 54C65, 03E17.

The authors were partially supported by the EU Research and Training Network HPRN-CT2002-00287, United States-Israel BSF Grant 2002323, and the Koshland Center for Basic Research, respectively. 
(2) Scheepers property 25] if for each sequence $\left\{\mathcal{U}_{n}\right\}_{n \in \mathbb{N}}$ of open covers of $X$, there exist finite sets $\mathcal{F}_{n} \subseteq \mathcal{U}_{n}, n \in \mathbb{N}$, such that for each finite set $F \subseteq X$, there is $n$ such that $F \subseteq \bigcup_{U \in \mathcal{F}_{n}} U$.

(3) Hurewicz property [12, 13] if for each sequence $\left\{\mathcal{U}_{n}\right\}_{n \in \mathbb{N}}$ of open covers of $X$, there exist finite sets $\mathcal{F}_{n} \subseteq \mathcal{U}_{n}, n \in \mathbb{N}$, such that for each element $x \in X$, $x \in \bigcup_{U \in \mathcal{F}_{n}} U$ for all but finitely many $n$.

(4) Rothberger property [24] if for each sequence $\left\{\mathcal{U}_{n}\right\}_{n \in \mathbb{N}}$ of open covers of $X$, there exist elements $U_{n} \in \mathcal{U}_{n}, n \in \mathbb{N}$, such that $X=\bigcup_{n \in \mathbb{N}} U_{n}$.

Except for the second, all of these properties are classical. They share the same structure and can be defined in a unified manner [25, 14]. These properties were analyzed in many papers and form an active area of mathematical research; see [26, 16, 29, 6] and the references therein.

The relations between the mentioned group theoretic and general topological properties is thoroughly investigated in [19, 32. Here, we consider only the group theoretic properties. Clearly, the group theoretic properties are related as follows:

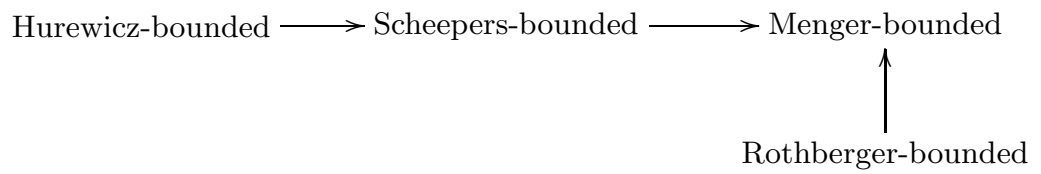

Babinkostova [1] proved that a metrizable group $G$ is Hurewicz-bounded if, and only if, $G$ is a subgroup of a $\sigma$-compact group (see [19]). Neither the leftmost horizontal implication nor the vertical implication can be inverted - even when restricting our attention to metrizable groups. The question as to whether the remaining implication can be inverted remained thus far open [5, 2, 6, 19].

Problem 3. Is every Menger-bounded group Scheepers-bounded?

The notions of Menger-bounded and Scheepers-bounded groups are related in the following elegant manner. For each $k$, let $G^{k}$ be the direct product of $k$ copies of $G$.

Theorem 4 (Babinkostova-Kočinac-Scheepers [2]). G is Scheepers-bounded if, and only if, $G^{k}$ is Menger-bounded for all $k$.

In light of Theorem 4. Problem 3 asks whether there could be a (metrizable) group $G$ such that for some $k, G^{k}$ is Menger-bounded but $G^{k+1}$ is not. The proof of Theorem [4 in 2] actually shows that the following holds for each natural number $k$. Since this is used in the sequel, we give a proof.

Lemma 5. $G^{k}$ is Menger-bounded if, and only if, for each sequence $\left\{U_{n}\right\}_{n \in \mathbb{N}}$ of neighborhoods of the unit of $G$ there exist finite sets $F_{n} \subseteq G, n \in \mathbb{N}$, such that for each $F \subseteq G$ with $|F|=k$ there is $n$ such that $F \subseteq F_{n} \cdot U_{n}$.

Proof. $(\Rightarrow)$ Let $U_{n}, n \in \mathbb{N}$, be neighborhoods of the unit of $G$. Then $U_{n}{ }^{k}, n \in \mathbb{N}$, are neighborhoods of the unit of $G^{k}$. Take finite $G_{n} \subseteq G^{k}$ such that $G^{k}=\bigcup_{n} G_{n} \cdot U_{n}{ }^{k}$. Adding elements if necessary, we may assume that each $G_{n}$ has the form $F_{n}{ }^{k}$ for some finite $F_{n} \subseteq G$. The sets $F_{n}$ are as required: Given $g_{1}, \ldots, g_{k} \in G$, there is $n$ such that $\left(g_{1}, \ldots, g_{k}\right) \in F_{n}{ }^{k} \cdot U_{n}{ }^{k}=\left(F_{n} \cdot U_{n}\right)^{k}$, and therefore $g_{1}, \ldots, g_{k} \in F_{n} \cdot U_{n}$.

$(\Leftarrow)$ It suffices to consider basic neighborhoods of the unit of $G^{k}$. Let $V_{n}=$ $U_{n, 1} \times \cdots \times U_{n, k}, n \in \mathbb{N}$, be such that each $U_{n, i}$ is a neighborhood of the unit of $G$. For each $n, U_{n}=U_{n, 1} \cap \cdots \cap U_{n, k}$ is a neighborhood of the unit of $G$. Take 
finite $F_{n} \subseteq G, n \in \mathbb{N}$, such that for each $F \subseteq G$ with $|F|=k$, there is $n$ such that $F \subseteq F_{n} \cdot V_{n}$. Given $\left(g_{1}, \ldots, g_{k}\right) \in G^{k}$, take $F=\left\{g_{1}, \ldots, g_{k}\right\}$. If needed, add elements to $F$ to have $|F|=k$. Then, whenever $F \subseteq F_{n} \cdot U_{n}$, we have that $\left(g_{1}, \ldots, g_{k}\right) \in F^{k} \subseteq\left(F_{n} \cdot U_{n}\right)^{k} \subseteq F_{n}{ }^{k} \cdot V_{n}$. Thus, the finite sets $F_{n}{ }^{k}, n \in \mathbb{N}$, are as required for the Menger-boundedness of $G^{k}$.

We give a negative answer to Problem 3 by showing that, assuming the Continuum Hypothesis or just a portion of it, there is for each $k$ a metrizable group $G$ such that $G^{k}$ is Menger-bounded but $G^{k+1}$ is not.

Some special hypothesis is necessary in order to prove such a result: Banakh and Zdomskyy [7, 6, and later (independently) Mildenberger and Shelah [23, proved that, consistently, every topological group with Menger-bounded square is Scheepers-bounded.

Question 1 of Banakh, Nickolas, and Sanchis [5] asks whether each Mengerbounded subgroup of $\mathbb{C}^{\mathbb{N}}$ (with coordinate-wise addition) is mixable or $o_{\mathcal{F}}$-bounded for some filter $\mathcal{F}$. As it is proved there that mixable Menger-bounded groups are Scheepers bounded, and the same holds for groups which are $o_{\mathcal{F}}$-bounded for some filter $\mathcal{F}$, we obtain a negative answer to both questions: The groups we construct are, in particular, subgroups of $\mathbb{C}^{\mathbb{N}}$.

The problem of whether, consistently, every Menger-bounded group is Scheepersbounded is yet to be addressed. The answer to this problem is positive if, and only if, the answer to the following problem is positive.

Problem 6. Is it consistent that for each Menger-bounded group $G, G^{2}$ is Mengerbounded?

There seems to be no straightforward negative answer to Problem [6] If $G$ is abelian and Menger-bounded but $G^{2}$ is not, then $G$ cannot be analytic, and it cannot be a free topological group over a Tychonoff space, either [6, 33].

\section{Specializing the question for the Baer-Specker Group}

The Baer-Specker group is the abelian group $\left(\mathbb{Z}^{\mathbb{N}},+\right)$ where + denotes coordinatewise addition. Subgroups of the Baer-Specker group form a rich source of examples of groups with various boundedness properties [3, 27, 9, 19, 32. The advantage of working in $\mathbb{Z}^{\mathbb{N}}$ is that the boundedness properties there can be stated in a purely combinatorial manner.

We use mainly self-evident notation. The quantifiers $\left(\exists^{\infty} n\right)$ and $\left(\forall^{\infty} n\right)$ stand for "there exist infinitely many $n$ " and "for all but finitely many $n$ ", respectively. The canonical basis for the topology of $\mathbb{Z}^{\mathbb{N}}$ consists of the sets

$$
[s]=\left\{f \in \mathbb{Z}^{\mathbb{N}}: s \subseteq f\right\},
$$

where $s$ ranges over all finite sequences of integers. For natural numbers $k<m$, $[k, m)=\{k, k+1, \ldots, m-1\}$. For a partial function $f: \mathbb{N} \rightarrow \mathbb{Z},|f|$ is the function with the same domain, which satisfies $|f|(n)=|f(n)|$, where in this case $|\cdot|$ denotes the absolute value. For partial functions $f, g: \mathbb{N} \rightarrow \mathbb{N}$ with $\operatorname{dom}(f) \subseteq \operatorname{dom}(g)$, $f \leq g$ means: For each $n$ in the domain of $f, f(n) \leq g(n)$. Similarly, $f \leq k$ means: For each $n$ in the domain of $f, f(n) \leq k$. Finally, for a set $X$ and $k \in \mathbb{N}$, $[X]^{k}=\{F \subseteq X:|F|=k\}$.

In a manner similar to the characterizations given in [19], we prove the following. 
Theorem 7. Assume that $G$ is a subgroup of $\mathbb{Z}^{\mathbb{N}}$. The following conditions are equivalent:

(1) $G^{k}$ is Menger-bounded.

(2) For each increasing $h \in \mathbb{N}^{\mathbb{N}}$, there is $f \in \mathbb{N}^{\mathbb{N}}$ such that

$$
\left(\forall F \in[G]^{k}\right)(\exists n)(\forall g \in F)|g| \uparrow[0, h(n)) \leq f(n) .
$$

(3) For each increasing $h \in \mathbb{N}^{\mathbb{N}}$, there is $f \in \mathbb{N}^{\mathbb{N}}$ such that

$$
\left(\forall F \in[G]^{k}\right)\left(\exists^{\infty} n\right)(\forall g \in F)|g| \uparrow[0, h(n)) \leq f(n) .
$$

(4) There is $f \in \mathbb{N}^{\mathbb{N}}$ such that

$$
\left(\forall F \in[G]^{k}\right)\left(\exists^{\infty} n\right)(\forall g \in F)|g| \uparrow[0, n) \leq f(n) .
$$

Proof. $(1 \Rightarrow 2)$ Fix an increasing $h \in \mathbb{N}^{\mathbb{N}}$. For each $n$, take $U_{n}=[0 \uparrow[0, h(n))]$. Using Lemma 5, find finite $F_{n} \subseteq G, n \in \mathbb{N}$, such that each $k$-element subset of $G$ is contained in $F_{n}+U_{n}$ for some $n$. Define $f \in \mathbb{N}^{\mathbb{N}}$ by

$$
f(n)=\max \left\{|a(i)|: a \in F_{n} \text { and } i<h(n)\right\}
$$

for each $n$. Fix $F \in[G]^{k}$. Take $n$ such that $F \subseteq F_{n}+U_{n}$. For each $g \in F$, there is $a \in F_{n}$ such that $g \in a+U_{n}=[a \uparrow[0, h(n))]$; that is, $g\lceil[0, h(n))=a \uparrow[0, h(n))$, and therefore $|g| \uparrow[0, h(n))=|a| \uparrow[0, h(n)) \leq f(n)$.

$(2 \Rightarrow 1)$ Assume that $\left\{U_{n}\right\}_{n \in \mathbb{N}}$ is a sequence of neighborhoods of 0 in $\mathbb{Z}^{\mathbb{N}}$. Take an increasing $h \in \mathbb{N}^{\mathbb{N}}$ such that $[0 \uparrow[0, h(n))] \subseteq U_{n}$ for each $n$. Apply (2) for $h$ to obtain $f$. For each $n$ and each $s \in \mathbb{Z}^{[0, h(n))}$ with $|s| \leq f$, choose (if possible) $a_{s} \in G$ such that $a_{s} \uparrow[0, h(n))=s$. If this is impossible, take $a_{s}=0$. Let $F_{n}=\left\{a_{s}: s \in \mathbb{Z}^{[0, h(n))},|s| \leq f\right\}$. We claim that the sets $F_{n}$ are as required in Lemma 5. Given $F \in[G]^{k}$, let $n$ be such that $|g| \uparrow[0, h(n)) \leq f(n)$ for each $g \in F$. Then for each $g \in F$, there is $s \in \mathbb{Z}^{[0, h(n))}$ such that $g \uparrow[0, h(n))=s=a_{s} \uparrow[0, h(n))$, and thus

$$
g \in\left[a_{s} \uparrow[0, h(n))\right]=a_{s}+[0 \uparrow[0, h(n))] \subseteq a_{s}+U_{n} \subseteq F_{n}+U_{n} .
$$

$(1 \Rightarrow 3)$ Let $\mathbb{N}=\bigcup_{m} I_{m}$ be a partition into infinite sets. Fix $m$. For each $n \in I_{m}$, take $U_{n}=[0 \uparrow[0, h(n))]$. The arguments of $(1 \Rightarrow 2)$ show that there is $f_{m}: I_{m} \rightarrow$ $\mathbb{N}$ such that for each $F \in[G]^{k}$, there is $n \in I_{m}$ such that $|g| \uparrow[0, h(n)) \leq f_{m}(n)$ for all $g \in F$. Take $f=\bigcup_{m} f_{m}$.

$(3 \Rightarrow 2)$ and $(3 \Rightarrow 4)$ are trivial.

$(4 \Rightarrow 3)$ This was pointed out by Banakh and Zdomskyy, and later independently by Simon. Indeed, fix any increasing $h \in \mathbb{N}^{\mathbb{N}}$. Let $f$ be as in (4). We may assume that $f$ is increasing. Define $\tilde{f}(n)=f(h(n+1))$ for each $n$. Fix $F \in[G]^{k}$.

$$
I=\{n: n>h(0) \text { and }(\forall g \in F)|g| \uparrow[0, n) \leq f(n)\}
$$

is infinite. For each such $n \in I$, let $m$ be such that $n \in[h(m), h(m+1))$. Then for each $g \in F$,

$$
|g| \uparrow[0, h(m)) \leq|g| \uparrow[0, n) \leq f(n) \leq f(h(m+1))=\tilde{f}(m) .
$$

As $I$ is infinite, there are infinitely many such $m$. 


\section{An important COROLLARY OF THE MAIN THEOREM}

The purpose of this section is twofold: Making a significant corollary of our main result (Theorem 11) accessible to a wider audience, and exposing the reader to the technically delicate proof of Theorem 11 via a more accessible proof. Readers who are experienced with cardinal characteristics of the continuum may, however, wish to try moving directly to the next section, which is essentially self-contained.

Theorem $8(\mathrm{CH})$. There is a Menger-bounded group $G \leq \mathbb{Z}^{\mathbb{N}}$ such that $G^{2}$ is not Menger-bounded.

We first give an informal outline of the proof. Assume the Continuum Hypothesis. By transfinite induction on $\alpha<\aleph_{1}$, we will choose generators $g_{0}^{\alpha}, g_{1}^{\alpha} \in \mathbb{Z}^{\mathbb{N}}$ and let $G \leq \mathbb{Z}^{\mathbb{N}}$ be the group generated by $\left\{g_{0}^{\alpha}, g_{1}^{\alpha}: \alpha<\aleph_{1}\right\}$.

Enumerate $\mathbb{Z}^{2}=\left\{\left(a_{n}, b_{n}\right): n \in \mathbb{N}\right\}$ with each pair $(a, b)$ occurring infinitely often, and enumerate $\mathbb{Z}^{\mathbb{N}}=\left\{d_{\alpha}: \alpha<\aleph_{1}\right\}$. At step $\alpha$, let $M_{\alpha} \leq \mathbb{Z}^{\mathbb{N}}$ contain all functions encountered in earlier steps, as well as $d_{\alpha}$, and assume that $M_{\alpha}$ is closed under all operations required in the proof. $M_{\alpha}$ is countable, and we choose $h_{\alpha} \in \mathbb{N}^{\mathbb{N}}$ which grows much faster than any element of $M_{\alpha}$. Fix $n$. We choose a solution of $a_{n} x+b_{n} y=0$ over $\mathbb{Z}$ with $\max \{|x|,|y|\} \geq d_{\alpha}\left(h_{\alpha}(n+1)\right)$, but not greater than necessary (henceforth: minimal solution). For each $k \in[h(n), h(n+1))$, we set $\left(g_{0}^{\alpha}(k), g_{1}^{\alpha}(k)\right)=(x, y)$.

The fact that $\max \left\{\left|g_{0}^{\alpha}\left(h_{\alpha}(n)\right)\right|,\left|g_{1}^{\alpha}\left(h_{\alpha}(n)\right)\right|\right\} \geq d_{\alpha}\left(h_{\alpha}(n+1)\right)$ guarantees that $G^{2}$ is not Menger-bounded (using Theorem $7(4)$ ).

The proof that $G$ is Menger-bounded is more subtle (a preservation argument). A general element of $G$ is a linear combination

$$
g=r_{1} g_{0}^{\alpha_{1}}+t_{1} g_{1}^{\alpha_{1}}+\cdots+r_{M} g_{0}^{\alpha_{M}}+t_{M} g_{1}^{\alpha_{M}}
$$

over $\mathbb{Z}$, for some $\alpha_{1}<\cdots<\alpha_{M}<\aleph_{1}$. Consider the partial sum $r_{1} g_{0}^{\alpha_{1}}+t_{1} g_{1}^{\alpha_{1}}$. The minimal solution function belongs to $M_{\alpha_{1}}$, and using the fact that $h_{\alpha_{1}}$ increases much faster than members of $M_{\alpha_{1}}$, we find infinitely many $j$ such that

$$
\left|r_{1} g_{0}^{\alpha_{1}}+t_{1} g_{1}^{\alpha_{1}}\right| \uparrow[0, j) \leq j .
$$

By induction, we assume that for infinitely many $j$, the $m-1$-st partial sum satisfies

$$
\left|r_{1} g_{0}^{\alpha_{1}}+t_{1} g_{1}^{\alpha_{1}}+\cdots+r_{m-1} g_{0}^{\alpha_{m-1}}+t_{m-1} g^{\alpha_{m-1}}\right| \uparrow[0, j) \leq c j,
$$

where $c$ is some constant, and prove the same assertion for the $m$-th partial sum. The set of $j$-s which satisfy (1) defines a function which belongs to $M_{\alpha_{m-1}}$, and consequently almost each interval $\left[h_{\alpha_{m}}(n), h_{\alpha_{m}}(n+1)\right)$ contains such a $j$. Take $n$ such that $\left(a_{n}, b_{n}\right)=\left(r_{m}, t_{m}\right)$, and take $j$ satisfying (1). On the interval $\left[h_{\alpha_{m}}(n), j\right)$, $r_{m} g_{0}^{\alpha_{m}}+t_{m} g_{1}^{\alpha_{m}}$ is 0 , and thus the $m$-th partial sum is equal to the $m-1$-st partial sum, and the same bound $c j$ applies on that interval. To take care of $\left[0, h_{\alpha_{m}}(n)\right)$, we modify the above argument so that $h_{\alpha_{m}}(n+1) \leq j$, and use the fact that $h_{\alpha_{m}}(n)$ is much smaller than $h_{\alpha_{m}}(n+1)$ in a direct calculation.

At the end, there will be infinitely many $j$ such that the $M$-th partial sum, which is equal to $g$, will be bounded on $[0, j)$ by some constant multiple of $j$, which is bounded, for example, by $j^{2}$.

Proof of Theorem 8 . Fix a partition of $\mathbb{N}$ into infinitely many infinite sets $I_{l}, l \in \mathbb{N}$. Replacing each $I_{l}$ with the set $\left\{2 n, 2 n+1: n \in I_{l}\right\}$, we may assume that for each even $n, n \in I_{l}$ if, and only if, $n+1 \in I_{l}$. Enumerate $\mathbb{Z}^{2}$ as $\left\{\left(a_{n}, b_{n}\right): n \in \mathbb{N}\right\}$, 
such that the sequence $\left\{\left(a_{n}, b_{n}\right)\right\}_{n \in I_{l}}$ is constant for each $l$. Fix an enumeration $\left\{d_{\alpha}: \alpha<\aleph_{1}\right\}$ of all increasing members of $\mathbb{N}^{\mathbb{N}}$.

We carry out a construction by induction on $\alpha<\aleph_{1}$. Step $\alpha$ : For each $m$, take a solution to the homogeneous linear equation $a_{m} x+b_{m} y=0$ over $\mathbb{Q}$. Multiplying $(x, y)$ by a large enough integer multiple of the common denominator of $x$ and $y$, we may assume that $x, y \in \mathbb{Z}$ and $\max \{|x|,|y|\} \geq N$ for any prescribed $N$. Using that, define nondecreasing functions $\varphi_{\alpha, m} \in \mathbb{N}^{\mathbb{N}}, m \in \mathbb{N}$, by

$$
\varphi_{\alpha, m}(n)=\min \left\{\max \{|x|,|y|\}: \begin{array}{l}
x, y \in \mathbb{Z}, a_{m} x+b_{m} y=0, \\
\max \{|x|,|y|\} \geq d_{\alpha}(n)
\end{array}\right\}
$$

and consequently define $\varphi_{\alpha} \in \mathbb{N}^{\mathbb{N}}$ by

$$
\varphi_{\alpha}(n)=\max \left\{\varphi_{\alpha, m}(n): m \leq n\right\} .
$$

Let $M_{\alpha} \subseteq \mathbb{Z}^{\mathbb{N}}$ be the smallest set (with respect to inclusion) containing $\varphi_{\alpha}$ and all functions defined in stages $<\alpha$, and such that $M_{\alpha}$ is closed under all operations relevant for the proof. For example, closing $M_{\alpha}$ under the following operations suffices:

(a) $g(n) \mapsto \hat{g}(n)=\max \{|g(m)|: m \leq n\}$;

(b) for each $c \in \mathbb{N}: g(n) \mapsto g_{<c}(n)=\min \{j: n \leq j, g(j)<c \cdot(j+1)\}$, whenever $g_{<c}$ is well-defined;

(c) $(f, g) \mapsto f+g$;

(d) $g \mapsto-g$.

((c)+(d) mean that $M_{\alpha} \leq \mathbb{Z}^{\mathbb{N}}$.)

By induction, $M_{\alpha}$ is countable. Take an increasing $h_{\alpha} \in \mathbb{N}^{\mathbb{N}}$ such that for each $f \in M_{\alpha}$,

$$
f\left(h_{\alpha}(n)\right)<h_{\alpha}(n+1)
$$

for all but finitely many $n 1$

Define $g_{0}^{\alpha}, g_{1}^{\alpha} \in \mathbb{Z}^{\mathbb{N}}$ as follows: For each $n$, choose $c, d \in \mathbb{Z}^{2}$ as in the definition of $\varphi_{\alpha, n}\left(h_{\alpha}(n+1)\right)$, and define $\left(g_{0}^{\alpha}\left(h_{\alpha}(n)\right), g_{1}^{\alpha}\left(h_{\alpha}(n)\right)\right)=(c, d)$ so that for all $n$,

$$
\begin{aligned}
a_{n} g_{0}^{\alpha}\left(h_{\alpha}(n)\right)+b_{n} g_{1}^{\alpha}\left(h_{\alpha}(n)\right) & =0, \text { and } \\
\max \left\{\left|g_{0}^{\alpha}\left(h_{\alpha}(n)\right)\right|,\left|g_{1}^{\alpha}\left(h_{\alpha}(n)\right)\right|\right\} & =\varphi_{\alpha, n}\left(h_{\alpha}(n+1)\right) \geq d_{\alpha}\left(h_{\alpha}(n+1)\right) .
\end{aligned}
$$

The remaining values of the functions $g_{i}^{\alpha}$ are defined by declaring these functions constant on each interval $\left[h_{\alpha}(n), h_{\alpha}(n+1)\right)$.

Take the generated subgroup $G=\left\langle g_{0}^{\alpha}, g_{1}^{\alpha}: \alpha<\aleph_{1}\right\rangle$ of $\mathbb{Z}^{\mathbb{N}}$. We will show that $G$ is as required in the theorem.

$G^{2}$ is not Menger-bounded. Let $f \in \mathbb{N}^{\mathbb{N}}$. Take $\alpha<\aleph_{1}$ such that $f(n)<d_{\alpha}(n)$ for all $n$. For each $m$, let $n$ be such that $m-1 \in\left[h_{\alpha}(n), h_{\alpha}(n+1)\right)$. As each function $g_{i}^{\alpha}$ is constant on the interval $\left[h_{\alpha}(n), h_{\alpha}(n+1)\right)$, we have by (2) that

$$
\begin{aligned}
& \max \left\{\left|g_{0}^{\alpha}(m-1)\right|,\left|g_{1}^{\alpha}(m-1)\right|\right\} \\
& \quad=\max \left\{\left|g_{0}^{\alpha}\left(h_{\alpha}(n)\right)\right|,\left|g_{1}^{\alpha}\left(h_{\alpha}(n)\right)\right|\right\} \geq d_{\alpha}\left(h_{\alpha}(n+1)\right) \geq d_{\alpha}(m)>f(m) .
\end{aligned}
$$

This violates Theorem 7 (4) for $k=2$.

\footnotetext{
${ }^{1}$ To achieve that, enumerate $M_{\alpha} \cap \mathbb{N}^{\mathbb{N}}=\left\{f_{n}: n \in \mathbb{N}\right\}$, define $h_{\alpha}(0)=0$, and inductively for each $n>0$, define $h_{\alpha}(n+1)=\max \left\{h_{\alpha}(n), f_{0}\left(h_{\alpha}(n)\right), \ldots, f_{n}\left(h_{\alpha}(n)\right)\right\}+1$.
} 
$G$ is Menger-bounded. Take $f(n)=n^{2}$. We will prove that $f$ is as required in Theorem $7(4)$.

Fix $g \in G$. Then there are $M \in \mathbb{N}, \alpha_{1}<\cdots<\alpha_{M}<\aleph_{1}$, and integers $r_{1}, t_{1}, \ldots, r_{M}, t_{M}$ such that

$$
g=r_{1} g_{0}^{\alpha_{1}}+t_{1} g_{1}^{\alpha_{1}}+\cdots+r_{M} g_{0}^{\alpha_{M}}+t_{M} g_{1}^{\alpha_{M}} .
$$

Let $g_{0}=0$, and for each $m=1, \ldots, M$ define

$$
g_{m}=r_{1} g_{0}^{\alpha_{1}}+t_{1} g_{1}^{\alpha_{1}}+\cdots+r_{m} g_{0}^{\alpha_{m}}+t_{m} g_{1}^{\alpha_{m}} .
$$

We prove, by induction on $m=0, \ldots, M$, that for an appropriate constant $c_{m}$, we have (using the notation in (a) on page 1756) that

$$
\hat{g}_{m}(j) \leq c_{m} \cdot(j+1)
$$

for infinitely many $j$.

The case $m=0$ is trivial. We show how to move from $m-1$ to $m$. Assume that

$$
J_{m-1}=\left\{j: \hat{g}_{m-1}(j) \leq c_{m-1} \cdot(j+1)\right\}
$$

is infinite.

By (2), for each $n>0$,

$$
\begin{aligned}
& \max \left\{\left|g_{0}^{\alpha_{m}}\left(h_{\alpha_{m}}(n-1)\right)\right|,\left|g_{1}^{\alpha_{m}}\left(h_{\alpha_{m}}(n-1)\right)\right|\right\} \\
& \quad=\varphi_{\alpha_{m}, n-1}\left(h_{\alpha_{m}}(n)\right) \leq \varphi_{\alpha_{m}}\left(h_{\alpha_{m}}(n)\right) .
\end{aligned}
$$

As $\varphi_{\alpha_{m}}$ and $h_{\alpha_{m}}$ are nondecreasing,

$$
\max \left\{\hat{g}_{0}^{\alpha_{m}}\left(h_{\alpha_{m}}(n-1)\right), \hat{g}_{1}^{\alpha_{m}}\left(h_{\alpha_{m}}(n-1)\right)\right\} \leq \varphi_{\alpha_{m}}\left(h_{\alpha_{m}}(n)\right),
$$

and since $\varphi_{\alpha_{m}} \in M_{\alpha_{m}}$

$$
\begin{aligned}
& \max \left\{\hat{g}_{0}^{\alpha_{m}}\left(h_{\alpha_{m}}(n-1)\right), \hat{g}_{1}^{\alpha_{m}}\left(h_{\alpha_{m}}(n-1)\right)\right\} \\
& \quad \leq \varphi_{\alpha_{m}}\left(h_{\alpha_{m}}(n)\right)<h_{\alpha_{m}}(n+1)
\end{aligned}
$$

for all but finitely many $n$.

As $\alpha_{1}, \ldots, \alpha_{m-1}<\alpha_{m}$ and $M_{\alpha_{m}} \leq \mathbb{Z}^{\mathbb{N}}, g_{m-1} \in M_{\alpha_{m}}$. Thus, $\tilde{g}=\hat{g}_{m-1} \in$ $M_{\alpha_{m}}$. As $J_{m-1}$ is infinite, we have (using the notation of (b) on page 3) that $\tilde{g}_{<c_{m-1}}(n)=\min \left\{j: n \leq j \in J_{m-1}\right\}$ is well defined, and $\tilde{g}_{<c_{m-1}} \in M_{\alpha_{m}}$. Consequently, $\tilde{g}_{<c_{m-1}}\left(h_{\alpha_{m}}(n+1)\right)<h_{\alpha_{m}}(n+2)$ for all but finitely many $n$. In other words, for each large enough $n$, there is $j \in J_{m-1}$ such that

$$
h_{\alpha_{m}}(n+1) \leq j<h_{\alpha_{m}}(n+2) .
$$

Let $l$ be such that for each $n \in I_{l},\left(a_{n}, b_{n}\right)=\left(r_{m}, t_{m}\right)$. For each large enough even $n \in I_{l},\left(a_{n}, b_{n}\right)=\left(a_{n+1}, b_{n+1}\right)=\left(r_{m}, t_{m}\right)$, and thus by (2)),

$$
\begin{aligned}
r_{m} g_{0}^{\alpha_{m}}\left(h_{\alpha_{m}}(n)\right)+t_{m} g_{1}^{\alpha_{m}}\left(h_{\alpha_{m}}(n)\right) & =0, \\
r_{m} g_{0}^{\alpha_{m}}\left(h_{\alpha_{m}}(n+1)\right)+t_{m} g_{1}^{\alpha_{m}}\left(h_{\alpha_{m}}(n+1)\right) & =0 .
\end{aligned}
$$

By (3),

$$
g_{m} \uparrow\left[h_{\alpha_{m}}(n), h_{\alpha_{m}}(n+2)\right)=g_{m-1}\left\lceil\left[h_{\alpha_{m}}(n), h_{\alpha_{m}}(n+2)\right) .\right.
$$

Fix $j$ as in (5). Let $p \in[0, j+1)$. 
Case 1: $p \geq h_{\alpha_{m}}(n)$. As $j<h_{\alpha_{m}}(n+2)$,

$$
\left[h_{\alpha_{m}}(n), j+1\right) \subseteq\left[h_{\alpha_{m}}(n), h_{\alpha_{m}}(n+2)\right),
$$

and by (6) and the membership $j \in J_{m-1}$,

$$
\left|g_{m}(p)\right|=\left|g_{m-1}(p)\right| \leq \hat{g}_{m-1}(j) \leq c_{m-1} \cdot(j+1) .
$$

Case 2: $p<h_{\alpha_{m}}(n)$. By the definition of $g_{m}$,

$$
\left|g_{m}(p)\right| \leq\left|g_{m-1}(p)\right|+2 \max \left\{\left|r_{m}\right|,\left|t_{m}\right|\right\} \cdot \max \left\{\left|g_{0}^{\alpha_{m}}(p)\right|,\left|g_{1}^{\alpha_{m}}(p)\right|\right\} .
$$

As $p<h_{\alpha_{m}}(n) \leq j \in J_{m-1},\left|g_{m-1}(p)\right| \leq \hat{g}_{m-1}(j) \leq c_{m-1} \cdot(j+1)$. Using $p \leq$ $h_{\alpha_{m}}(n)-1$, (4), and $h_{\alpha_{m}}(n+1) \leq j$, we obtain

$$
\left|g_{i}^{\alpha_{m}}(p)\right| \leq \hat{g}_{i}^{\alpha_{m}}\left(h_{\alpha_{m}}(n)-1\right)=\hat{g}_{i}^{\alpha_{m}}\left(h_{\alpha_{m}}(n-1)\right)<h_{\alpha_{m}}(n+1) \leq j
$$

for each $i=0,1$. Together with (7), we have that

$$
\begin{aligned}
\left|g_{m}(p)\right| & \leq\left|g_{m-1}(p)\right|+2 \max \left\{\left|r_{m}\right|,\left|t_{m}\right|\right\} \cdot \max \left\{\left|g_{0}^{\alpha_{m}}(p)\right|,\left|g_{1}^{\alpha_{m}}(p)\right|\right\} \\
& \leq c_{m-1} \cdot(j+1)+2 \max \left\{\left|r_{m}\right|,\left|t_{m}\right|\right\} j \\
& \leq c_{m-1} \cdot(j+1)+2 \max \left\{\left|r_{m}\right|,\left|t_{m}\right|\right\} \cdot(j+1) \\
& =\left(c_{m-1}+2 \max \left\{\left|r_{m}\right|,\left|t_{m}\right|\right\}\right) \cdot(j+1) .
\end{aligned}
$$

Take $c_{m}=c_{m-1}+2 \max \left\{\left|r_{m}\right|,\left|t_{m}\right|\right\}$.

We have proved that for almost all even $n \in I_{l}$ there is

$$
j \in\left[h_{\alpha_{m}}(n+1), h_{\alpha_{m}}(n+2)\right)
$$

such that $j \in J_{m}$. There are infinitely many even $n \in I_{l}$, and therefore $J_{m}$ is infinite. This completes the inductive proof.

Now, for each $j$ in the infinite set $J_{M}$ such that $c_{M} \leq j$,

$$
|g| \uparrow[0, j+1) \leq \hat{g}(j) \leq c_{M} \cdot(j+1) \leq(j+1)^{2}=f(j+1) .
$$

By Theorem $7, G$ is Menger-bounded.

It is rather straightforward to extend the above proof to get for each $k$, a group $G \leq \mathbb{Z}^{\mathbb{N}}$ such that $G^{k}$ is Menger-bounded, but $G^{k+1}$ is not. To see that, have a quick look at the proof of Theorem 11 .

\section{THE MAIN THEOREM}

Our main Theorem[11 requires a weak portion of the Continuum Hypothesis, that is best stated in terms of cardinal characteristics of the continuum. An excellent introduction to the topic is 8 . However, we give a self-contained treatment.

For $f, g \in \mathbb{N}^{\mathbb{N}}, f \leq^{*} g$ means $f(n) \leq g(n)$ for all but finitely many $n$. A subset $Y$ of $\mathbb{N}^{\mathbb{N}}$ is bounded if there is $g \in \mathbb{N}^{\mathbb{N}}$ such that $f \leq^{*} g$ for all $f \in Y$. At the other extreme, a subset $Y$ of $\mathbb{N}^{\mathbb{N}}$ is dominating if for each $f \in \mathbb{N}^{\mathbb{N}}$ there is $g \in Y$ such that $f \leq^{*} g$.

$\mathfrak{b}$ is the minimal cardinality of an unbounded subset of $\mathbb{N}^{\mathbb{N}}$, and $\mathfrak{d}$ is the minimal cardinality of a dominating subset of $\mathbb{N}^{\mathbb{N}}$. An argument as in footnote 1 shows that $\aleph_{1} \leq \mathfrak{b}$. Thus, $\aleph_{1} \leq \mathfrak{b} \leq \mathfrak{d} \leq 2^{\aleph_{0}}$. The hypothesis $\mathfrak{b}=\mathfrak{d}$ is strictly weaker than the Continuum Hypothesis [8]. By inspection, one can see that for the proof of Theorem 8 , it suffices to assume that $\mathfrak{b}=\mathfrak{d}$. To extend this observation further, we introduce the following new cardinal characteristics. 
Definition 9. Fix a partition $\mathcal{P}=\left\{I_{l}: l \in \mathbb{N}\right\}$ of $\mathbb{N}$ such that for each $l$, there are infinitely many $n$ such that $n, n+1 \in I_{l}$. For $f \in \mathbb{N}^{\mathbb{N}}$ and an increasing $h \in \mathbb{N}^{\mathbb{N}}$, write

$$
[f \ll h]=\{n: f(h(n))<h(n+1)\} .
$$

$\mathfrak{d}^{\prime}(\mathcal{P})$ is the cardinal such that the following are equivalent:

(1) $\kappa<\mathfrak{d}^{\prime}(\mathcal{P})$.

(2) For each $Y \subseteq \mathbb{N}^{\mathbb{N}}$ such that $|Y|=\kappa$, there is an increasing $h \in \mathbb{N}^{\mathbb{N}}$ such that for each $f \in Y$,

$$
(\forall l)\left(\exists^{\infty} n\right) n, n+1 \in I_{l} \cap[f \ll h] .
$$

Clearly, $\mathfrak{b} \leq \mathfrak{d}^{\prime}(\mathcal{P}) \leq \mathfrak{d}$ for each $\mathcal{P}$. We first point out that the hypothesis "there is $\mathcal{P}$ such that $\mathfrak{d}^{\prime}(\mathcal{P})=\mathfrak{d}$ " is strictly weaker than the hypothesis $\mathfrak{b}=\mathfrak{d}$. Let $\operatorname{cov}(\mathcal{M})$ be the minimal cardinality of a cover of $\mathbb{N}^{\mathbb{N}}$ by meager (first category) sets. It is consistent that $\mathfrak{b}<\operatorname{cov}(\mathcal{M})=\mathfrak{d}[\underline{8}$.

Lemma 10. For each $\mathcal{P}, \operatorname{cov}(\mathcal{M}) \leq \mathfrak{d}^{\prime}(\mathcal{P})$.

Proof. Fix a partition $\mathcal{P}=\left\{I_{l}: l \in \mathbb{N}\right\}$ of $\mathbb{N}$ such that for each $l$, there are infinitely many $n$ such that $n, n+1 \in I_{l}$. Let $\mathbb{N}^{\uparrow \mathbb{N}}$ be the set of all increasing elements of $\mathbb{N}^{\mathbb{N}}$. $\mathbb{N}^{\uparrow \mathbb{N}}$ is homeomorphic to $\mathbb{N}^{\mathbb{N}}$. It therefore suffices to find a cover of $\mathbb{N}^{\uparrow \mathbb{N}}$ by $\mathfrak{d}^{\prime}(\mathcal{P})$ many nowhere-dense subsets of $\mathbb{N}^{\uparrow \mathbb{N}}$.

Take $Y \subseteq \mathbb{N}^{\mathbb{N}}$ such that $|Y|=\mathfrak{d}^{\prime}(\mathcal{P})$ and such that Definition 9(2) fails for $Y$. That is: For each $h \in \mathbb{N}^{\uparrow \mathbb{N}}$, there are $f \in Y$ and $l$ such that

$$
\left(\forall^{\infty} n\right) n, n+1 \in I_{l} \rightarrow f(h(n)) \geq h(n+1) \text { or } f(h(n+1)) \geq h(n+2) .
$$

For $f \in Y$ and $l, m \in \mathbb{N}$, let

$$
Y_{f, l, m}=\left\{h \in \mathbb{N}^{\uparrow \mathbb{N}}: \begin{array}{l}
(\forall n \geq m) n, n+1 \in I_{l} \\
\rightarrow f(h(n)) \geq h(n+1) \text { or } f(h(n+1)) \geq h(n+2)
\end{array}\right\} .
$$

$Y_{f, l, m}$ is nowhere dense in $\mathbb{N}^{\uparrow} \mathbb{N}$ : Given $k$ and an increasing finite sequence $s \in \mathbb{N}^{k}$, let $n \geq \max \{k, m\}$ be such that $n, n+1 \in I_{l}$. Let $\tilde{s}$ be an extension of $s$ to an increasing sequence of length $n+3$ such that $f(\tilde{s}(n))<\tilde{s}(n+1)$ and $f(\tilde{s}(n+1))<\tilde{s}(n+2)$. Then $Y_{f, l, m} \cap[\tilde{s}]=\emptyset$.

As $\bigcup\left\{Y_{f, l, m}: f \in Y, l, m \in \mathbb{N}\right\}=\mathbb{N}^{\uparrow \mathbb{N}}, \operatorname{cov}(\mathcal{M}) \leq \mathfrak{d}^{\prime}(\mathcal{P}) \cdot \aleph_{0}=\mathfrak{d}^{\prime}(\mathcal{P})$. 22 .

A more thorough analysis of the cardinals $\mathfrak{d}^{\prime}(\mathcal{P})$ is carried out by Mildenberger

Theorem 11. Assume that there is $\mathcal{P}$ such that $\mathfrak{d}^{\prime}(\mathcal{P})=\mathfrak{d}$. Then for each $k$, there is a group $G \leq \mathbb{Z}^{\mathbb{N}}$ such that $G^{k}$ is Menger-bounded but $G^{k+1}$ is not Mengerbounded.

Proof. Fix a partition $\mathcal{P}=\left\{I_{l}: l \in \mathbb{N}\right\}$ of $\mathbb{N}$ such that for each $l$, there are infinitely many $n$ such that $n, n+1 \in I_{l}$, and such that $\mathfrak{d}^{\prime}(\mathcal{P})=\mathfrak{d}$.

Enumerate $\mathbb{Z}^{k \times(k+1)}$ as $\left\{A_{n}: n \in \mathbb{N}\right\}$, such that the sequence $\left\{A_{n}\right\}_{n \in I_{l}}$ is constant for each $l$. Fix a dominating family of increasing functions $\left\{d_{\alpha}: \alpha<\mathfrak{d}\right\} \subseteq \mathbb{N}^{\mathbb{N}}$. For $v=\left(v_{0}, \ldots, v_{k}\right) \in \mathbb{Z}^{k+1}$, write $\|v\|$ or $\left\|v_{0}, \ldots, v_{k}\right\|$ for $\max \left\{\left|v_{0}\right|, \ldots,\left|v_{k}\right|\right\}$ (the supremum norm of $v$ ). 
We carry out a construction by induction on $\alpha<\mathfrak{d}$. Step $\alpha$ : Define functions $\varphi_{\alpha, m} \in \mathbb{N}^{\mathbb{N}}, m \in \mathbb{N}$, by

$$
\varphi_{\alpha, m}(n)=\min \left\{\|v\|: v \in \mathbb{Z}^{k+1},\|v\| \geq d_{\alpha}(n), A_{m} v=\overrightarrow{0}\right\} .
$$

Also, define $\varphi_{\alpha} \in \mathbb{N}^{\mathbb{N}}$ by

$$
\varphi_{\alpha}(n)=\max \left\{\varphi_{\alpha, m}(n): m \leq n\right\} .
$$

Let $M_{\alpha} \subseteq \mathbb{Z}^{\mathbb{N}}$ be the smallest set (with respect to inclusion) containing $\varphi_{\alpha}$ and all functions defined in stages $<\alpha$, and such that $M_{\alpha}$ is closed under all operations relevant for the proof. For example, closing $M_{\alpha}$ under the following operations suffices:

(a) $g(n) \mapsto \hat{g}(n)=\max \{|g(m)|: m \leq n\}$;

(b) $(g(n), f(n)) \mapsto \max \{|g(n)|,|f(n)|\}$;

(c) for each $c \in \mathbb{N}: g(n) \mapsto g_{<c}(n)=\min \{j: n \leq j, g(j)<c \cdot(j+1)\}$, whenever $g_{<c}$ is well-defined;

(d) $(f, g) \mapsto f+g$;

(e) $g \mapsto-g$.

There are countably many such operations, and by induction, $\left|M_{\alpha}\right| \leq \max \left\{\aleph_{0},|\alpha|\right\}$ $<\mathfrak{d}=\mathfrak{d}^{\prime}(\mathcal{P})$. By the definition of $\mathfrak{d}^{\prime}(\mathcal{P})$, there is an increasing $h_{\alpha} \in \mathbb{N}^{\mathbb{N}}$ such that for each $f \in M_{\alpha} \cap \mathbb{N}^{\mathbb{N}}$,

$$
(\forall l)\left(\exists^{\infty} n\right) n, n+1 \in I_{l} \cap\left[f \ll h_{\alpha}\right] .
$$

Define $k+1$ elements $g_{0}^{\alpha}, \ldots, g_{k}^{\alpha} \in \mathbb{Z}^{\mathbb{N}}$ as follows: For each $n$, let $v \in \mathbb{Z}^{k+1}$ be a witness for the definition of $\varphi_{\alpha, n}\left(h_{\alpha}(n+1)\right)$; namely,

$$
\begin{aligned}
\varphi_{\alpha, n}\left(h_{\alpha}(n+1)\right) & =\|v\| \geq d_{\alpha}\left(h_{\alpha}(n+1)\right), \\
A_{n} v & =\overrightarrow{0},
\end{aligned}
$$

and define

$$
\left(\begin{array}{c}
g_{0}^{\alpha}\left(h_{\alpha}(n)\right) \\
\vdots \\
g_{k}^{\alpha}\left(h_{\alpha}(n)\right)
\end{array}\right)=v
$$

so that

$$
A_{n} \cdot\left(\begin{array}{c}
g_{0}^{\alpha}\left(h_{\alpha}(n)\right) \\
\vdots \\
g_{k}^{\alpha}\left(h_{\alpha}(n)\right)
\end{array}\right)=\overrightarrow{0} .
$$

The remaining values of the functions $g_{i}^{\alpha}$ are defined by declaring these functions constant on each interval $\left[h_{\alpha}(n), h_{\alpha}(n+1)\right)$. By (10) and (12),

$$
\left\|g_{0}^{\alpha}\left(h_{\alpha}(n)\right), \ldots, g_{k}^{\alpha}\left(h_{\alpha}(n)\right)\right\|=\varphi_{\alpha, n}\left(h_{\alpha}(n+1)\right)
$$

for all $n$.

Take the generated subgroup $G=\left\langle g_{0}^{\alpha}, \ldots, g_{k}^{\alpha}: \alpha<\mathfrak{d}\right\rangle$ of $\mathbb{Z}^{\mathbb{N}}$. We will show that $G$ is as required in the theorem. 
$G^{k+1}$ is not Menger-bounded. We use Theorem 7. Let $f \in \mathbb{N}^{\mathbb{N}}$. Take $\alpha<\mathfrak{d}$ such that $f<^{*} d_{\alpha}$, and set $F=\left\{g_{0}^{\alpha}, \ldots, g_{k}^{\alpha}\right\} \in[G]^{k+1}$. For each large enough $m$, $f(m)<d_{\alpha}(m)$. Fix such an $m$. Let $n$ be such that $m-1 \in\left[h_{\alpha}(n), h_{\alpha}(n+1)\right)$. As each function $g_{i}^{\alpha}$ is constant on the interval $\left[h_{\alpha}(n), h_{\alpha}(n+1)\right)$, and using (13) and (10), we have that

$$
\begin{aligned}
& \left\|g_{0}^{\alpha}(m-1), \ldots, g_{k}^{\alpha}(m-1)\right\| \\
& \quad=\left\|g_{0}^{\alpha}\left(h_{\alpha}(n)\right), \ldots, g_{k}^{\alpha}\left(h_{\alpha}(n)\right)\right\|=\varphi_{\alpha, n}\left(h_{\alpha}(n+1)\right) \\
& \quad \geq d_{\alpha}\left(h_{\alpha}(n+1)\right) \geq d_{\alpha}(m)>f(m) .
\end{aligned}
$$

This violates Theorem $7(4)$ for the power $k+1$.

$G^{k}$ is Menger-bounded. Take $f(n)=n^{2}$. Clearly, $f$ dominates all functions $f_{c}(n)=$ $c \cdot n, c \in \mathbb{N}$. We will prove that $f$ is as required in Theorem [7(4).

Fix $F=\left\{g_{0}, \ldots, g_{k-1}\right\} \subseteq G$. Then there are $M \in \mathbb{N}, \alpha_{1}<\cdots<\alpha_{M}<\mathfrak{d}$, and matrices $B_{1}, \ldots, B_{M} \in \mathbb{Z}^{k \times(k+1)}$ such that

$$
\left(\begin{array}{c}
g_{0} \\
\vdots \\
g_{k-1}
\end{array}\right)=B_{1}\left(\begin{array}{c}
g_{0}^{\alpha_{1}} \\
\vdots \\
g_{k}^{\alpha_{1}}
\end{array}\right)+\cdots+B_{M}\left(\begin{array}{c}
g_{0}^{\alpha_{M}} \\
\vdots \\
g_{k}^{\alpha_{M}}
\end{array}\right) .
$$

Let $g_{0,0}=\cdots=g_{k-1,0}=0$, and for each $m=1, \ldots, M$ let

$$
\left(\begin{array}{c}
g_{0, m} \\
\vdots \\
g_{k-1, m}
\end{array}\right)=B_{1}\left(\begin{array}{c}
g_{0}^{\alpha_{1}} \\
\vdots \\
g_{k}^{\alpha_{1}}
\end{array}\right)+\cdots+B_{m}\left(\begin{array}{c}
g_{0}^{\alpha_{m}} \\
\vdots \\
g_{k}^{\alpha_{m}}
\end{array}\right) .
$$

We prove, by induction on $m=0, \ldots, M$, that for an appropriate constant $c_{m}$, there are infinitely many $j$ such that

$$
\left\|\hat{g}_{0, m}(j), \ldots, \hat{g}_{k-1, m}(j)\right\| \leq c_{m} \cdot(j+1) .
$$

By the definition of $f$, this suffices.

The case $m=0$ is trivial. We show how to move from $m-1$ to $m$. Assume that

$$
J_{m-1}=\left\{j:\left\|\hat{g}_{0, m-1}(j), \ldots, \hat{g}_{k-1, m-1}(j)\right\| \leq c_{m-1} \cdot(j+1)\right\}
$$

is infinite.

As $\alpha_{1}, \ldots, \alpha_{m-1}<\alpha_{m}$, we have by (14) that $g_{0, m-1}, \ldots, g_{k-1, m-1} \in M_{\alpha_{m}}$. By (a), (b), (c), the functions

$$
g(n)=\left\|\hat{g}_{0, m-1}(n), \ldots, \hat{g}_{k-1, m-1}(n)\right\|
$$

and $g_{<c_{m-1}}$ both belong to $M_{\alpha_{m}}$. Note that

$$
g_{<c_{m-1}}(n)=\min \left\{j: n \leq j \in J_{m-1}\right\}
$$

and is therefore well defined. Thus, $\max \left\{g_{<c_{m-1}}, \varphi_{\alpha_{m}}\right\} \in M_{\alpha_{m}}$.

For each $i \leq k$ and each $n>0$, as $n-1 \leq h_{\alpha_{m}}(n)$, we have by (13) that

$$
\left|g_{i}^{\alpha_{m}}\left(h_{\alpha_{m}}(n-1)\right)\right| \leq \varphi_{\alpha_{m}, n-1}\left(h_{\alpha_{m}}(n)\right) \leq \varphi_{\alpha_{m}}\left(h_{\alpha_{m}}(n)\right) .
$$

As $\varphi_{\alpha_{m}}$ and $h_{\alpha_{m}}$ are nondecreasing,

$$
\left\|\hat{g}_{0}^{\alpha_{m}}\left(h_{\alpha_{m}}(n-1)\right), \ldots, \hat{g}_{k}^{\alpha_{m}}\left(h_{\alpha_{m}}(n-1)\right)\right\| \leq \varphi_{\alpha_{m}}\left(h_{\alpha_{m}}(n)\right) .
$$


Thus, if $l$ is such that for each $n \in I_{l}, A_{n}=B_{m}$, we have by (15) and (16) that

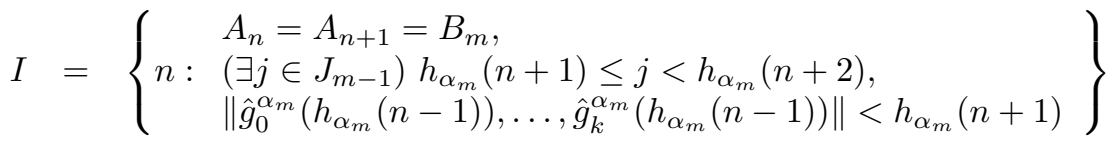

$$
\begin{aligned}
& \supseteq\left\{\begin{array}{ll}
n: n+1 \in I_{l}, \\
g_{<c_{m-1}}\left(h_{\alpha_{m}}(n+1)\right)<h_{\alpha_{m}}(n+2), \\
\varphi_{\alpha_{m}}\left(h_{\alpha_{m}}(n)\right)<h_{\alpha_{m}}(n+1)
\end{array}\right\} \\
& \supseteq\left\{n: n, n+1 \in I_{l} \cap\left[\max \left\{g_{<c_{m-1}}, \varphi_{\alpha_{m}}\right\} \ll h_{\alpha_{m}}\right]\right\} \text {. }
\end{aligned}
$$

As $\max \left\{g_{<c_{m-1}}, \varphi_{\alpha_{m}}\right\} \in M_{\alpha_{m}}$, we have by the definition of $h_{\alpha_{m}}$ (9) that the last set is infinite, and therefore so is $I$.

Let $n \in I$. Then $A_{n}=A_{n+1}=B_{m}$, and thus by (11) and (12),

$$
B_{m} \cdot\left(\begin{array}{c}
g_{0}^{\alpha_{m}}\left(h_{\alpha_{m}}(n)\right) \\
\vdots \\
g_{k}^{\alpha_{m}}\left(h_{\alpha_{m}}(n)\right)
\end{array}\right)=B_{m} \cdot\left(\begin{array}{c}
g_{0}^{\alpha_{m}}\left(h_{\alpha_{m}}(n+1)\right) \\
\vdots \\
g_{k}^{\alpha_{m}}\left(h_{\alpha_{m}}(n+1)\right)
\end{array}\right)=\overrightarrow{0} .
$$

By (14), for each $i<k$,

$$
g_{i, m}\left\lceil\left[h_{\alpha_{m}}(n), h_{\alpha_{m}}(n+2)\right)=g_{i, m-1}\left\lceil\left[h_{\alpha_{m}}(n), h_{\alpha_{m}}(n+2)\right) .\right.\right.
$$

As $n \in I$, there is $j \in J_{m-1}$ such that $h_{\alpha_{m}}(n+1) \leq j<h_{\alpha_{m}}(n+2)$, and

$$
\left\|\hat{g}_{0}^{\alpha_{m}}\left(h_{\alpha_{m}}(n-1)\right), \ldots, \hat{g}_{k}^{\alpha_{m}}\left(h_{\alpha_{m}}(n-1)\right)\right\|<h_{\alpha_{m}}(n+1) .
$$

Let $p \in[0, j+1)$.

Case 1: $p \geq h_{\alpha_{m}}(n)$. As $j<h_{\alpha_{m}}(n+2)$,

$$
\left[h_{\alpha_{m}}(n), j+1\right) \subseteq\left[h_{\alpha_{m}}(n), h_{\alpha_{m}}(n+2)\right),
$$

and by (17) and the membership $j \in J_{m-1}$,

$$
\left|g_{i, m}(p)\right|=\left|g_{i, m-1}(p)\right| \leq \hat{g}_{i, m-1}(j) \leq c_{m-1} \cdot(j+1)
$$

for all $i<k$.

Case 2: $p<h_{\alpha_{m}}(n)$. Let $C$ be the maximal absolute value of a coordinate of $B_{m}$. For all $i<k$, by the definition of $g_{i, m}$,

$$
\left|g_{i, m}(p)\right| \leq\left|g_{i, m-1}(p)\right|+(k+1) C \cdot \max \left\{\left|g_{i}^{\alpha_{m}}(p)\right|: i \leq k\right\} .
$$

As $p<h_{\alpha_{m}}(n) \leq j \in J_{m-1},\left|g_{i, m-1}(p)\right| \leq \hat{g}_{i, m-1}(j) \leq c_{m-1} \cdot(j+1)$. Using $p \leq h_{\alpha_{m}}(n)-1$, (18), $g_{i}^{\alpha_{m}}$ being constant on $\left[h_{\alpha_{m}}(n-1), h_{\alpha_{m}}(n)\right)$ and $h_{\alpha_{m}}(n+1) \leq j$, we obtain

$$
\left|g_{i}^{\alpha_{m}}(p)\right| \leq \hat{g}_{i}^{\alpha_{m}}\left(h_{\alpha_{m}}(n)-1\right)=\hat{g}_{i}^{\alpha_{m}}\left(h_{\alpha_{m}}(n-1)\right)<h_{\alpha_{m}}(n+1) \leq j
$$

for each $i \leq k$. Together with (19), we have that

$$
\begin{aligned}
\left|g_{i, m}(p)\right| & \leq\left|g_{i, m-1}(p)\right|+(k+1) C \cdot \max \left\{\left|g_{i}^{\alpha_{m}}(p)\right|: i \leq k\right\} \\
& \leq c_{m-1} \cdot(j+1)+(k+1) C j \\
& \leq c_{m-1} \cdot(j+1)+(k+1) C \cdot(j+1) \\
& =\left(c_{m-1}+(k+1) C\right) \cdot(j+1) .
\end{aligned}
$$

Take $c_{m}=c_{m-1}+(k+1) C$. We have proved that for each $n \in I$ there is $j \in$ $\left[h_{\alpha_{m}}(n+1), h_{\alpha_{m}}(n+2)\right)$ such that $j \in J_{m} . I$ is infinite, and therefore so is $J_{m}$. This completes the inductive proof, and consequently the proof of Theorem 11 
Remark 12. Mildenberger has recently proved that our assumption in Theorem[1] can be weakened to $\mathfrak{d} \leq \mathfrak{r}[22$.

\section{ACKNOWLEDGEMENTS}

We thank Heike Mildenberger and Lyubomyr Zdomskyy for their useful comments. Following the suggestions of the referee, we have substantially expanded the introductory and motivational parts of this paper.

\section{REFERENCES}

[1] L. Babinkostova, Metrizable groups and strict o-boundedness, Matematicki Vesnik 58 (2006), 131-138. MR2318228 (2008b:54055)

[2] L. Babinkostova, Lj. D.R. Kočinac, and M. Scheepers, Combinatorics of open covers (XI): Menger- and Rothberger-bounded groups, Topology and its Applications 154 (2007), 1269 1280. MR2310460 (2008g:54051)

[3] R. Baer, Abelian groups without elements of finite order, Duke Mathematical Journal 3 (1937), 68-122. MR.1545974

[4] T. Banakh, On index of total boundedness of (strictly) o-bounded groups, Topology and its Applications 120 (2002), 427-439. MR.1897272 (2003c:22003)

[5] T. Banakh, P. Nickolas, and M. Sanchis, Filter games and pathological subgroups of a countable product of lines, Journal of the Australian Mathematical Society 81 (2006), 321-350. MR2300160 (2008c:54024)

[6] T. Banakh and L. Zdomsky, Selection principles and infinite games on multicovered spaces and their applications, book in progress.

[7] T. Banakh and L. Zdomskyy, Selection principles and infinite games on multicovered spaces in: Selection Principles and Covering Properties in Topology (Lj. D.R. Kočinac, ed.), Quaderni di Matematica 18 (2006), Seconda Universita di Napoli, Caserta, 2-51. MR 2395750 (2009g:54047)

[8] A. R. Blass, Combinatorial cardinal characteristics of the continuum, in: Handbook of Set Theory (M. Foreman, A. Kanamori, and M. Magidor, eds.), Kluwer Academic Publishers, Dordrecht, to appear.

[9] A. R. Blass, Abelian Group Theory papers, http://www.math.lsa.umich.edu/ ablass/abgp. html

[10] C. Hernandez, Topological groups close to being $\sigma$-compact, Topology and its Applications 102 (2000), 101-111. MR 1739266 (2000k:54032)

[11] C. Hernandez, D. Robbie and M. Tkačenko, Some properties of o-bounded and strictly obounded groups, Applied General Topology 1 (2000), 29-43. MR1796930 (2001g:22002)

[12] W. Hurewicz, Über eine Verallgemeinerung des Borelschen Theorems, Mathematische Zeitschrift 24 (1925), 401-421.

[13] W. Hurewicz, Über Folgen stetiger Funktionen, Fundamenta Mathematicae 9 (1927), 193204.

[14] W. Just, A. Miller, M. Scheepers, and P. Szeptycki, The combinatorics of open covers. II, Topology and its Applications 73 (1996), 241-266. MR.1419798 (98g:03115a)

[15] L. Kočinac, Selection principles in uniform spaces, Note di Matematica 22 (2003), 127-139. MR2112735 (2006b:54019)

[16] L. Kocinac, Selected results on selection principles, in: Proceedings of the 3rd Seminar on Geometry and Topology (Sh. Rezapour, ed.), July 15-17, Tabriz, Iran, 2004, 71-104. MR2090207 (2005g:54038)

[17] A. Krawczyk and H. Michalewski, Linear metric spaces close to being $\sigma$-compact, Technical Report 46 (2001) of the Institute of Mathematics, Warsaw University. www.minuw.edu.pl/ english/research/reports/tr-imat/46/products.ps

[18] A. Krawczyk and H. Michalewski, An example of a topological group, Topology and its Applications 127 (2003), 325-330. MR.1941171 (2003j:54034)

[19] M. Machura and B. Tsaban, The combinatorics of the Baer-Specker group, Israel Journal of Mathematics, 168 (2008), 125-151. MR2448054(2009g:20116)

[20] K. Menger, Einige Überdeckungssätze der Punktmengenlehre, Sitzungsberichte der Wiener Akademie 133 (1924), 421-444. 
[21] H. Michalewski, Function spaces with topology of pointwise convergence, doctoral dissertation, Faculty of Mathematics, Informatics and Mechanic, Warsaw University (2003).

[22] H. Mildenberger, Cardinal characteristics for Menger-bounded subgroups, Topology and its Applications 156 (2008), 130-137. MR2463833 (2009i:03049)

[23] H. Mildenberger and S. Shelah, Menger-bounded subgroups of the Baer-Speker group, unpublished notes.

[24] F. Rothberger, Eine Verschärfung der Eigenschaft C, Fundamenta Mathematicae 30 (1938), $50-55$.

[25] M. Scheepers, Combinatorics of open covers I: Ramsey theory, Topology and its Applications 69 (1996), 31-62. MR:1378387(97h:90123)

[26] M. Scheepers, Selection principles and covering properties in topology, Note di Matematica 22 (2003), 3-41. MR2112729 (2005h:54024)

[27] E. Specker, Additive Gruppen von Folgen ganzer Zahlen, Portugaliae Mathematica 9 (1950), 131-140. MR0039719(12:587b)

[28] M. Tkačenko, Introduction to topological groups, Topology and its Applications 86 (1998), 179-231. MR.1623960 (99b:54064)

[29] B. Tsaban, Some new directions in infinite-combinatorial topology, in: Set Theory (J. Bagaria and S. Todorčevic, eds.), Trends in Mathematics, Birkhauser, 2006, 225-255. MR2267150 (2007f:03064)

[30] B. Tsaban, o-bounded groups and other topological groups with strong combinatorial properties, Proceedings of the American Mathematical Society 134 (2006), 881-891. MR2180906 (2006h:54034)

[31] B. Tsaban and L. Zdomskyy, Scales, fields, and a problem of Hurewicz, Journal of the European Mathematical Society (JEMS) 10 (2008), 837-866. MR.2421163

[32] T. Weiss, A note on unbounded strongly measure zero subgroups of the Baer-Specker group, Topology and its Applications 156 (2008), 138-141. MR2463834

[33] L. Zdomskyy, o-Boundedness of free objects over a Tychonoff space, Matematychni Studii 25 (2006), 10-28. MR2254996(2008a:54025)

Institute of Mathematics, University of Silesia, ul. Bankowa 14, 40-007 Katowice, Poland - And - Department of Mathematics, Bar-Ilan University, Ramat Gan 52900, ISRAEL

E-mail address: machura@ux2.math.us.edu.pl

Einstein Institute of Mathematics, The Hebrew University of Jerusalem, Givat Ram, 91904 Jerusalem, Israel - and - Department of Mathematics, Rutgers University, New Brunswick, Piscataway, New Jersey 08854

E-mail address: shelah@math.huji.ac.il

Department of Mathematics, Bar-Ilan University, Ramat-Gan 52900, Israel - and Department of Mathematics, Weizmann Institute of Science, Rehovot 76100, Israel

E-mail address: tsaban@math.biu.ac.il

$U R L:$ http://www.cs.biu.ac.il/ tsaban 